\title{
The Resemblance of an Autocorrelation Function to a Power Spectrum Density for a Spike Train of an Auditory Model
}

\author{
Yu. V. Ushakov*, A. A. Dubkov* and B. Spagnolo ${ }^{\dagger}$ \\ ${ }^{*}$ Lobachevsky State University, Radiophysics department, 23, Gagarin ave., Nizhniy Novgorod, \\ 603950, Russia.Contact e-mail: ushakov@rf.unn.ru. \\ ${ }^{\dagger}$ Dipartimento di Fisica, Group of Interdisciplinary Physics, Viale delle Scienze, Ed. 18, Universitá \\ di Palermo, I-90128, Italy
}

\begin{abstract}
In this work we develop an analytical approach for calculation of the all-order interspike interval density (AOISID), show its connection with the autocorrelation function, and try to explain the discovered resemblance of AOISID to the power spectrum of the same spike train.
\end{abstract}

Keywords: power spectrum, autocorrelation, inter-spike interval density

PACS: $87.19 .1 \mathrm{c}, 87.19 .11,43.80 .+\mathrm{p}$

Introduction. As it is well-known, the Fourier transformation allows imaging a signal as a sum of sinusoidal components. In case of a spike train, it seems to be not consequent to consider a sequence of sharp pulses as a sum of smooth sinusoids. Apparently, this is one of the reasons why neurophysiologists use the histogram of interspike intervals, in particular, all-order interspike interval density (AOISID), more often, then the power spectrum density (PSD). The power spectrum of a short in time pulse inevitably contains high frequency components, which do not have anything to do with interspike intervals. So, the spectrum provides just the redundant information about a pulse shape, which does not play a role in an inter-neuron communication. Actually, in the past, before the fast Fourier transformation algorithm invention and its wide applications, the usual tool for the signal analysis was a correlometer providing the autocorrelation function (ACF), which is stated [1] to be directly connected with AOISID.

In the presented work we describe a quite unexpected connection between ACF and PSD observed in the auditory system models. The connection between ACF and AOISID is also rigorously derived here.

The presented study has been motivated by the discovery of the resemblance of the PSD at the output of a simple neural model [2] to the AOISID at the output of much more complex model [1] of the same auditory system of mammals with similar parameters of input signals (Fig. 1A). The problem of the analysis of this resemblance was that we had analytical expressions for the PSD of the simple model, but did not have ones for the AOISID. As per the complex model, here we had only AOISID plots and a not clear enough statement about the direct correspondence between AOISID and ACF.

Model description. The mentioned PSD has been calculated for the model, which is described in details in the paper [2]. It consists of three Leaky Integrate-and-Fire neurons, two of which are the input (sensory) elements and are driven by sinusoidal signals, 


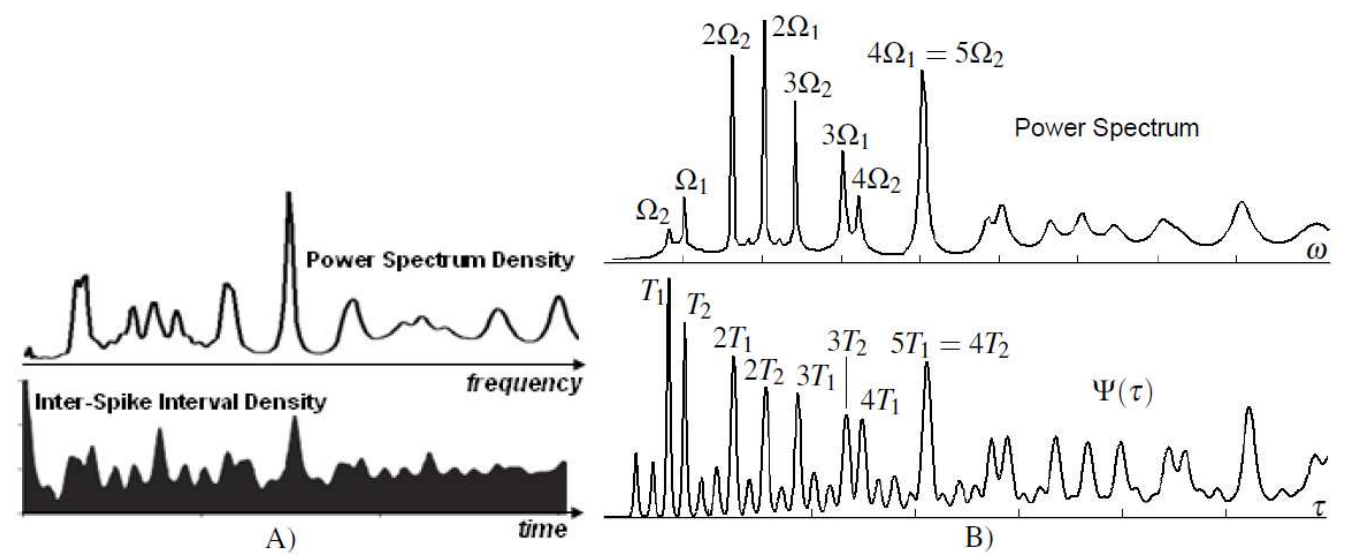

FIGURE 1. Resemblance of PSD to AOISID: A) PSD from Ref. [3] vs. AOISID from Ref. [1]; B) PSD and analytical AOISID from Eq. (2) for the same spike train.

and the third one is the output element receiving spikes from the sensory ones. Each neuron is also influenced by white Gaussian noise. In the previous studies, the characteristics of an output spike train of the model were analyzed at various combinations of input sinusoids' frequencies $\Omega_{1}, \Omega_{2}$, namely, the combinations, which are typical for harmonious and dissonant musical chords. It was shown $[2,3]$ that in a case of commensurable input frequencies, the system may be described by the hidden Markov chain with the finite number of states and the transition matrix $\left\{\pi_{i j}\right\}$. At the moment of the output spike generation, the system switches between the states. If the target $i^{\text {th }}$ state is known, then the distribution $\rho^{(i)}(t)$ of a time interval until the next output spike is also known. In the paper [3] the deriving procedure for the PSD formula is provided, given the matrix $\left\{\pi_{i j}\right\}$ and the distributions $\rho^{(i)}(t)$.

AOISID and ACF. In the paper [1], the following AOISID calculation procedure is proposed. First, all the first-order interspike intervals (ISIs) are extracted from the set of a number of parallel output spike trains. Then, the second-order ISIs are extracted, i.e., the sums of all pairs of consequent intervals. These second-order ISIs are added to the same array as the first-order ones. In the same way, the third-, fourth-, etc. order ISIs are collected in one place and then distributed into histogram bins. Despite the name of the characteristics, surely, this is not the "All-order" ISI distribution, because the maximal order of a considered ISI is always limited.

Let us consider some sequence of random intervals. The rigorous mathematical approach to the All-Order ISI Distribution requires understanding of the nature of the random quantity, which is being distributed. It is not hard to make sure that in our case the random quantity is the sum of random number $N$ of random intervals $t_{1}, t_{2}, t_{3}, \ldots$ :

$$
\tau=\sum_{n=1}^{N} t_{n}
$$

where $N \in\left\{1,2,3, \ldots, N_{\max }\right\}$ and $t_{n} \in(0,+\infty)$. For example, defining $N_{\max }=2$ and using the formula of the total probability, one can obtain the probability density of $\tau$ as $\Psi_{2}(t)=P\left(H_{1}\right) \rho_{1}(t)+P\left(H_{2}\right) \rho_{2}(t)$, where $H_{1}, H_{2}$ are the mutually exclusive events of having one or two addends in the sum Eq. (1), respectively; $P\left(H_{1}\right), P\left(H_{2}\right)$ are their 
probabilities; $\rho_{1}(t)$ is the probability density of one interspike interval $t_{1}$ to be in the delta-neighbourhood of $t$; and $\rho_{2}(t)$ is the same probability density for the sum of two consequent intervals $t_{1}+t_{2}$. Assuming $P\left(H_{1}\right)=P\left(H_{2}\right)$, what, at least, is not in contradiction with the procedure of Ref. [1], we obtain $\Psi_{2}(t)=\left[\rho_{1}(t)+\rho_{2}(t)\right] / 2$. In the general case, this formula allows inducing the following one:

$$
\Psi_{N_{\max }}(t)=\frac{1}{N_{\max }} \sum_{n=1}^{N_{\max }} \rho_{n}(t) .
$$

Here, the probability density $\rho_{2}(t)$ is calculated as the integral: $\int_{0}^{t} \rho\left(t_{1}, t-t_{1}\right) d t_{1}$, where $\rho\left(t_{1}, t_{2}\right)$ is the joint probability density for the consequent intervals $t_{1}$ and $t_{2}$. Analogously, $\rho_{3}(t)=\int_{0}^{t} d t_{1} \int_{0}^{t-t_{1}} \rho\left(t_{1}, t_{2}, t-t_{1}-t_{2}\right) d t_{2}$, etc.

In order to find a connection with the autocorrelation function, one should refer to the paper [4], where the following expression is proposed for ACF:

$$
K(\tau)=f^{2}\left[\delta(\tau)+\sum_{n=1}^{\infty} W_{t_{n}}(|\tau|)-1 / T\right] / T .
$$

Here, $f$ and $T$ are some constants; $\delta(t)$ is the Dirac delta-function; and the quantity $W_{t_{n}}(|\tau|)$ is just the same as $\rho_{n}(\tau)$ in the Eq. (2). Hence, one may assert the proportionality between ACF and AOISID, but not the identity.

AOISID and PSD. The problem of $\rho_{n}(t)$ calculation in the Eq. (2) has been solved in the aforementioned case (see "Model description") with the same approach as in Refs. [3, 5], resulting in the AOISID depicted in Fig. 1B. Obviously, there is a resemblance between the top panel and bottom panel plots. Actually, generation of input frequencies' multiples (here $\Omega_{1} / \Omega_{2}=5 / 4$ ) in the power spectrum of the output signal of a nonlinear system is a well-known phenomenon in physics. So, it is interesting to understand, why the temporal characteristics $\Psi(\tau)$ behaves in the same manner with respect to the periods $T_{1,2}=2 \pi / \Omega_{1,2}$ ? The other interesting question arises, if one remembers about the direct connection between ACF and PSD through the Fourier transformation. Indeed, is there any advantage in usage of a system with a signal having similar PSD and ACF? However, first of all: is this similarity typical for the brain subsystems, or this is just the negligible particular case? The theoretical research is in progress, and the experimental contribution is welcome and will be much appreciated.

Acknowledgements. The presented study is supported by the Russian Foundation for Basic Research (grant number 11-02-01418-a).

\section{REFERENCES}

1. P. Cariani, J. New Music Res. 30, 107-135 (2001).

2. Y. V. Ushakov, A. A. Dubkov, and B. Spagnolo, Phys. Rev. E 81, 041911-1-13 (2010).

3. Y. V. Ushakov, and A. A. Dubkov, Moscow University Physics Bulletin 65, 372-377 (2010).

4. O. A. Chichigina, A. A. Dubkov, D. Valenti, and B. Spagnolo, Phys. Rev. E 84, 021134 (2011).

5. Y. V. Ushakov, A. A. Dubkov, and B. Spagnolo, Phys. Rev. Lett. 107, 108103 (2011). 